Noname manuscript No.

(will be inserted by the editor)

\title{
The emergence of phase asynchrony and frequency modulation in metacommunities
}

\author{
Frederic Guichard \\ Yuxian Zhang \\ Frithjof Lutscher
}

Received: date / Accepted: date

\begin{abstract}
Spatial synchrony can summarize complex patterns of population abundance. Studies of phase synchrony predict that limited dispersal can drive either in-phase or out-of-phase synchrony, characterized by a constant phase difference among populations. We still lack an understanding of ecological processes leading to the loss of phase synchrony. Here we study the role of limited dispersal as a cause of phase asynchrony defined as fluctuating phase differences among populations. We adopt a minimal predator-prey model allowing for dispersal-induced phase asynchrony, and show its dependence on species traits. We show that phase asynchrony in a homogeneous metacommunity requires a minimum of three communities and is characterized by the emergence of regional frequency modulation of population fluctuations. This frequency modulation results in spectral signatures in local time series that can be used to infer the causes and properties of metacommunity dynamics. Dispersal-induced phase asynchrony extends the application of ecological theories of synchrony to non stationary time series, and predicts observed spatiotemporal patterns in marine metacommunities.
\end{abstract}

\footnotetext{
F. Guichard

Department of Biology, McGill University, 1205 Docteur Penfield, Montreal, Quebec H3A 1B1, Canada;

E-mail: frederic.guichard@mcgill.ca

Y. Zhang

Tianjin University, School of Mathematics, Tianjin, China.

F. Lutscher

Department of Mathematics and Statistics \& Department of Biology, Ottawa, Ontario, Canada.
} 
2

3

4

\section{Introduction}

A central challenge in ecology is to predict patterns of population abundance across spatial scales, especially for non-equilibrium dynamics. Predator-prey communities are known to exhibit stable cyclic dynamics, but our understanding of emerging patterns in metacommunities consisting of oscillating predatory-prey communities is still incomplete. The concept of spatial synchrony, the lack thereof, and the mechanisms of its emergence have provided an appropriate level of simplification to understand how spatiotemporal patterns affect population persistence (Abbott 2011), species coexistence (Lampert \& Hastings 2016), community stability (Koelle \& Vandermeer 2005) and ecosystem function (Marleau et al. 2014) over landscapes. Our work provides novel insights into drivers of asynchrony and identifies signatures in time series that distinguish these mechanisms from previously known ones.

In ecology, spatial synchrony can be measured as covariance among time series of population density (Koelle \& Vandermeer 2005, Lande et al. 1999, Liebhold et al. 2004). When time series contain periodic components (Turchin 2003), synchrony can also be studied by extracting their phase, i.e. the angular position of the variable along each cycle. Spatial synchrony can then be measured (Bjørnstad et al. 1999, Blasius et al. 1999) or predicted (Goldwyn \& Hastings 2007) as the phase difference among locations. Phase synchrony is defined as a constant phase difference among time series (i.e. phase locking) (Hoppensteadt \& Izhikevech 1997), which includes the special case of in-phase synchrony (zero phase difference). Phase synchrony is studied by contrasting in-phase (spatially homogeneous) to out-of-phase (spatially heterogeneous) phaselocked synchrony, and it has become central to the study of dispersal-driven heterogeneity in metapopulations and metacommunities (Blasius et al. 1999, Cazelles \& Boudjema 2001).

Ecological theories of synchrony predict that limited dispersal, predator movement, or correlated environmental variation can lock identical, distant communities into in- or out-of-phase synchrony (Liebhold et al. 2004, Bjørnstad et al. 1999). However, natural (Henden et al. 2009, Cazelles et al. 2008) and model (Jansen \& de Roos 2000) systems often fail to result in phase locking. For example, a marine metacommunity model for Mytilus californianus (California mussel) distribution over 1800km along the North East Pacific coast was used to simulate spatially coupled, identical locally oscillatory dynamics. The system did not phase lock but exhibited spatiotemporal variations in phase differences even though the coupling strength was in a regime where phase locking would be expected. These spatiotemporal patterns were used to validate the model against short-term (7 years) spatial data (Gouhier et al. 2010), but we still lack a general understanding of ecological processes causing deviations from in- or out-of-phase locking and non-stationary dynamics of phase difference.

With the above definition of phase synchrony (illustrated for two coupled communities in Fig 1A,B), we can define phase asynchrony as a deviation from phase locking. One form of phase asynchrony can arise from spatial heterogeneity when communities with different natural frequencies of local oscillations are (weakly) coupled and the heterogeneous frequencies persist 
(Fig 1C,D). This asynchrony can be synchronized by (sufficiently strong) dispersal (Koelle \& Vandermeer 2005, Blasius et al. 1999). A different form of phase asynchrony can arise when spatially heterogeneous environmental fluctuations modulate the frequencies of local population fluctuations (Allstadt et al. 2015) (Fig 1E,F). Phase asynchrony through the modulation of frequencies is a well known phenomenon in engineering and has been shown in chemical and physiological systems (Hoppensteadt \& Izhikevich 1998, Kim et al. 1998, Baesens et al. 1991, Emelianova et al. 2013). Here, we show that even in the absence of heterogeneous environmental variation, local dispersal can act as a driver of phase asynchrony by frequency modulation.

Our work extends current theories of ecological synchrony to dispersal-induced phase asynchrony. We study this phenomenon via a minimal dynamical metacommunity model consisting of three identical, spatially-coupled predator-prey systems. We use two complementary approaches: one based on the theory of phase equations for weakly coupled networks and bifurcation theory, the other based on numerical simulations of the dynamical equations, and guided by the bifurcation results. We also characterize statistical properties of time series of asynchronous metacommunities and reveal signatures of non-stationary (modulated) phase differences. We finally illustrate the robustness of frequency modulation in larger systems by using the marine metacommunity model mentioned above. Our model predicts the dependence of dispersal-induced asynchrony on species traits, and could provide a statistical signature of ecological processes across scales. The latter aspect is particularly important since inferring causes of asynchrony from time series can be challenging: phase asynchrony leads to non stationary time series and to spatial patterns that deviate from signatures of phase-locked systems such as regular traveling waves. Our results contribute to a more general ecological theory of synchrony, and in particular establish dispersal as a potential driver for spatial phase asynchrony, which has important consequences for rescue and compensatory effects between populations.

\section{Metacommunity model and phase equations}

We investigate spatial phase synchrony among weakly coupled predator-prey communities characterized by periodic fluctuations in their abundance. To study the stability of phase synchrony and the emergence of asynchrony, we consider three weakly coupled habitats as the minimum extension from previous studies on 2-patch metacommunities (Goldwyn \& Hastings 2007, Zhang et al. 2015). On patch $i$, we write the density of prey $\left(h_{i}\right)$ and predator $\left(p_{i}\right)$ as the vector $X_{i}=\left(h_{i}, p_{i}\right)^{T}$. We adopt the Rosenzweig-MacArthur model with logistic regulation of prey growth and saturating functional response of the predator. In nondimensional terms, the model on patch $i$ reads $d X_{i} / d t=F\left(X_{i}\right)$, where

$$
F\left(X_{i}\right)=\left(\frac{1}{\epsilon}\left(h_{i}\left(1-\alpha h_{i}\right)-\frac{h_{i} p_{i}}{1+h_{i}}\right), \frac{h_{i} p_{i}}{1+h_{i}}-\eta p_{i}\right)^{T} .
$$

72 Here, $\epsilon$ denotes a relative time scale of prey growth, $\alpha$ is the strength of prey-density regulation, and $\eta$ is the relative scale of predator death (for details please see Zhang et al. 2015). 
Finally, we assume that all three habitats are identical and that dispersal is well-mixed and linear (density-independent) among all three local communities. Then we study the following nondimensionalized model:

$$
\begin{aligned}
\frac{d X_{1}}{d t} & =F\left(X_{1}\right)+\delta\left(\frac{X_{2}}{2}+\frac{X_{3}}{2}-X_{1}\right), \\
\frac{d X_{2}}{d t} & =F\left(X_{2}\right)+\delta\left(\frac{X_{1}}{2}+\frac{X_{3}}{2}-X_{2}\right), \\
\frac{d X_{3}}{d t} & =F\left(X_{3}\right)+\delta\left(\frac{X_{1}}{2}+\frac{X_{2}}{2}-X_{3}\right),
\end{aligned}
$$

where $\delta$ denotes the (relative) time scale of dispersal and $F$ describes the local dynamics as above.

We study the question of synchrony or asynchrony in this model in two complementary ways: via direct numerical simulations of system (1) and via qualitative analysis of the corresponding phase-difference equations. To obtain the phase-difference equations from our model equations, we follow the theory for weakly connected networks (Hoppensteadt \& Izhikevech 1997) and previous work on predator-prey dynamics (Goldwyn \& Hastings 2007, Zhang et al. 2015). We assume that parameters for the uncoupled Rosenzweig-MacArthur system $d X / d t=F(X)$ on each patch are chosen such that there is an exponentially stable $T$-periodic solution $\gamma=\gamma(t) \subset$ $\mathbb{R}^{2}$. We denote the frequency of this orbit by $\Omega=2 \pi / T$.

We denote by $\phi_{i} \in[0,2 \pi), i=1,2,3$ the phase variable on the $i$-th patch. When the dispersal rate $\delta$ is small, the phase variables satisfy the following phase equations:

$$
\begin{aligned}
\frac{d \phi_{1}}{d t} & =\frac{1}{2} H\left(\phi_{2}-\phi_{1}\right)+\frac{1}{2} H\left(\phi_{3}-\phi_{1}\right), \\
\frac{d \phi_{2}}{d t} & =\frac{1}{2} H\left(\phi_{1}-\phi_{2}\right)+\frac{1}{2} H\left(\phi_{3}-\phi_{2}\right), \\
\frac{d \phi_{3}}{d t} & =\frac{1}{2} H\left(\phi_{1}-\phi_{3}\right)+\frac{1}{2} H\left(\phi_{2}-\phi_{3}\right),
\end{aligned}
$$

where the interaction function $H$ is given by

$$
H(x)=\frac{1}{T} \int_{0}^{T} \hat{\gamma}(t) \cdot(\gamma(t+x / \Omega)-\gamma(t)) d t .
$$

with $\hat{\gamma}(t)$ solving the system

$$
\begin{gathered}
\frac{d \hat{\gamma}(t)}{d t}=-\frac{D F(\gamma(t))^{T} \hat{\gamma}(t)}{} \hat{\gamma}(t) \cdot \gamma \prime(t)=1
\end{gathered}
$$

$D F$ is the derivative matrix of $\gamma(t)$ with respect to $X$, the superscript $T$ refers to the transpose of the matrix, and $\gamma(t)$ is the periodic solution on a single patch.

We can define two phase differences as $\psi_{i}:=\phi_{i+1}-\phi_{i}, i=1,2$ between patches $i$ and $i+1$. These variables satisfy the equations

$$
\begin{aligned}
\frac{d \psi_{1}}{d t} & =\frac{1}{2}\left[H\left(-\psi_{1}\right)+H\left(\psi_{2}\right)-H\left(\psi_{1}\right)-H\left(\psi_{1}+\psi_{2}\right)\right] \\
\frac{d \psi_{2}}{d t} & =\frac{1}{2}\left[H\left(-\psi_{1}-\psi_{2}\right)+H\left(-\psi_{2}\right)-H\left(-\psi_{1}\right)-H\left(\psi_{2}\right)\right] .
\end{aligned}
$$

\footnotetext{
These are the equations that we will study in detail via stability analysis.
} 


\section{Model Analysis}

We begin our model analysis with the phase-difference equations (6) and use the results as a guide for intensive numerical simulations of the full model system (1). We focus on $\eta$ as a bifurcation parameter for comparability with previous studies. We more specifically chose the range $0.15<\eta<0.4$ while all other parameters are fixed $(\epsilon=0.1, \alpha=0.4$, Fig 2$)$. Decreasing $\eta$ over that range contributes to a separation of temporal scales between predator and prey growth by decreasing predator mortality and/or increasing attack rate relative to prey growth. In a single patch, this separation leads to pulse-relaxation type oscillations (Goldwyn \& Hastings 2007). In a 2-patch system, pulse-relaxation oscillations are known to promote the emergence of bistability: stable out-of-phase and in-phase synchronous states coexist (Goldwyn \& Hastings 2007). We thus study how model parameters relating to traits of predators and prey, influence phase asynchrony in a 3-patch system.

\section{Phase-difference equations}

We summarize the most important results of our stability analysis of the phase difference equations (6) in the bifurcation diagram in Figure 2A. We explain the diagram and refer to the appendix for details and calculations as well as further illustrations.

The phase difference equations have $(0,0)$ as a steady state, representing the 'in-phase locked' scenario. This state exists independently of parameter values, and it is locally stable for the range of parameter values in our study. It is represented by the thick line at the bottom of the bifurcation diagram.

When exactly one of the phase differences is zero initially, it will remain zero for all times. By symmetry, the model has three of these '2-in-phase' states, independent of parameters, and they are unstable when the in-phase locked states are stable. We excluded them from Figure $2 \mathrm{~A}$ but show them in the detailed plots in the appendix.

The most important state in our study is that of equal phase differences between all three oscillators, sometimes called 'travelling wave state' (Goldwyn \& Hastings 2011) or 'rotating wave' (Ashwin et al. 1990). By symmetry, there are two of these states at $(2 \pi / 3,2 \pi / 3)$ and $(4 \pi / 3,4 \pi / 3)$. They are represented by the upper line in the bifurcation diagram in Figure 2A. These states are foci that can be stable (thick line) or unstable (thin line), depending on parameter values. The stability switch seems to occur via a Hopf bifurcation that can be subcritical (SH), where an unstable limit cycle surrounds a stable steady state, or supercritical $(\mathrm{H})$, where a stable limit cycle surrounds an unstable steady state. Such a limit cycle corresponds to sustained oscillations of the phase differences in our model and thereby to phase asynchrony. A limit cycle is represented in the bifurcation diagram only by the minimal phase difference along the orbit.

The bifurcation diagram shows a second scenario for the emergence of sustained phase oscillations. The scenario arises mathematically through a pair of saddle-node bifurcations of limit cycles (SNL) along the branch of the unstable limit cycle from the subcritical Hopf bifurcation. 
These bifurcations are extremely difficult to capture analytically, so that we provide only numerical evidence in the appendix. In such a bifurcation, two nested limit cycles, one stable and one unstable, collide and annihilate one another. In figure $2 \mathrm{~A}$, we see how a stable limit cycle (thick line) - corresponding to sustained phase oscillations - emerges in one saddle-node bifurcation with an unstable limit cycle (thin line) and disappears again in another such bifurcation.

In the next section, we explore how closely the full system (1) matches the results from the phase-difference system (6).

\section{Full model simulations}

We use results from the analysis of phase dynamics to guide our study of the stability of phase (a)synchrony and of its statistical properties in the full model system (1), thereby verifying that the phase-dynamics model is a good approximation to the phases of the full model.

We performed numerical simulations of the full system (1) using the ode 45 solver in Matlab (Mathworks). We ran 15 simulations until $t=200000$ for each set of parameter values and extracted the phase over the second half of each time series. As suggested by coexistence of stable objects in the phase difference equations (Fig 2A), initial conditions need to be chosen carefully in the basin of attraction to reveal these objects in simulations. Initial densities were semi-randomly sampled from the single-patch predator-prey limit-cycle for each set of parameter values, meaning that initial phase position along that orbit was constrained to be in the vicinity of one of 4 possible attractors: (i) $<1 \%$ deviation from all-in-phase (homogeneous phase locking), (ii) $<1 \%$ deviation from 2-in-phase (asymmetrical phase locking), (iii) $<1 \%$ deviation from equal, $2 \pi / 3$ phase differences (traveling wave phase-locked state), and (iv) a $<10 \%$ deviation from in-phase, and random initial phases for all 3 patches, in order to capture limit cycles in phase differences corresponding to phase asynchrony.

We extracted phases and calculated all local minima and maxima across pairwise phase difference time series following Cazelles \& Boudjema (2001). Local minima and maxima in phase differences reveal synchrony and asynchrony: if maxima are equal to minima of a time series then we have a locally stable phase-difference equilibrium (phase synchrony); if they differ, we have oscillatory dynamics of phase difference (phase asynchrony).

We first simulated the 3 -patch system across the same values of $\eta$ defined above, and for weak dispersal $\left(\delta=10^{-5}\right)$ in order to test for agreement between the full system and the analysis of phase equations (Fig 2A). For $\eta>0.3$, only the all-in-phase state (filled diamonds) is stable (Fig 2B). For $0.175<\eta<0.3$, the traveling wave states (red squares) also become locally stable, as predicted by the phase equations (Fig $2 \mathrm{~A}$, top thick line). For $\eta<0.2$, we see the appearance of a stable limit cycle of phase difference (asynchrony) through a saddle-node bifurcation of limit cycles (SNL) from initial conditions that are away from the in-phase and traveling wave states (filled circles). For $\eta<0.175$, the traveling wave state looses its local stability as indicated by the divergence between maximum and minimum phase differences (open red squares), and is replaced with asynchrony through a Hopf bifurcation. For $0.171<\eta<0.174$, two asynchronous 
orbits coexist, one resulting from the Hopf bifurcation of the traveling wave at $\eta \approx 0.174$, and one at $\eta \approx 0.2$ from the saddle-node bifurcation (Fig $2 \mathrm{~B}$ ). These results are compatible with the combination of a saddle-node and Hopf bifurcations obtained from phase equations (Fig 2A and Appendix), except for the brief coexistence $(0.171<\eta<0.174)$ between Hopf and saddle-node orbits that were only observed in numerical simulations of the full model.

Decreasing $\eta$ contributes to a separation of temporal scales between predator and prey growth. In a single patch, this separation leads to pulse-relaxation type oscillations. In a 2-patch system, pulse-relaxation oscillations are known to promote the emergence of bistability between out-ofphase and in-phase synchronous states (Goldwyn \& Hastings 2007). Scaling up to 3 patches, these same properties, when $\eta<0.2$ (Fig 2B) predict phase asynchrony, characterized by stable periodic oscillations of phase differences between each pair of coupled predator-prey communities (Fig 3B). For both 2- and 3-patch systems, the outcome is bistability: bistable (synchronous) steady states for 2 patches, and bistable steady state and orbit(s) for 3 patches. Bistability has been shown to be a general outcome for networks of homogeneous oscillators with symmetric (Ashwin et al. 1990) and asymmetric (Montbrió et al. 2004) coupling, where the generic bifurcation is a saddle-node, and the symmetry forces trajectories onto cycles. This property means that the traveling-wave among 3 patches can be interpreted as the analogue to the anti-phase synchrony between 2 patches. Coexistence of multiple stable states results in a dependence on initial conditions. However, for all $\eta$ values leading to asynchrony as one of the stable states, asynchrony was the only state reached from numerical simulations with random initial phases for all patches or from $10 \%$ deviation from the in-phase state. All other synchronous states (in-phase and traveling wave) are reached by constraining initial conditions to their vicinity.

Dispersal-induced asynchrony leads to non-stationary local and regional dynamics that are distinct from the expected effect of environmental heterogeneity. Instead of heterogeneous frequencies that would result in continuous growth of phase difference across habitats, dispersalinduced phase asynchrony is associated with oscillations of phase differences (Fig 2B) over much longer temporal scales than predator-prey cycles, as illustrated on Fig 3 for $\eta=0.15$ and $\delta=10^{-4}$. In contrast with the averaging effect of phase-locking, each pair of habitats goes through periods of apparent in-phase or out-of-phase synchrony (Fig 3B), and total metacommunity abundance periodically shifts between strong fluctuations during periods of weak phase differences, to periods of lower amplitude oscillations with transient frequency doubling (Fig $3 \mathrm{~A})$.

The non-stationary dynamics of phase differences results from an endogenous modulation of frequencies induced by dispersal (Fig 3C). Frequency modulation (Boashash 2016) explains non-stationary phase differences as the combination of 2 stationary signals: the 'carrier' signal (single-patch dynamics), and the lower frequency 'modulator' signal, which here emerges at the metacommunity level from spatial coupling. The magnitude of the modulator signal informs us about the maximum changes in frequency experienced by the carrier, while the modulator frequency sets the temporal scale of non-stationarity in phase differences. For example, carrier 
and modulator frequencies and amplitudes can be extracted from their time series (in Matlab), and for the parameter values used in Figures 3 and 4 , the frequency (measured in cycles $/ t$ ) of the carrier (oscillations from the single-patch dynamics) is $C=0.94$ (Fig 3A and 4A). Oscillations in the frequency of the time series correspond to the modulation signal and have frequency $M=$ 0.0007. The amplitude of that modulation signal then corresponds to the maximum modulation $D=0.0104$ (Fig 3C). The observed frequency modulation leads to phase asynchrony because frequencies are modulated with heterogeneous amplitudes (Fig 3C).

Frequency modulation also leaves signatures on the spectral properties of both local and regional time series. In a frequency domain, asynchronous time series reveal non-harmonic side bands (peaks at frequencies that are not integer multiples of the fundamental) around harmonic peaks (Fig 4B) that are missing from time series of single-patch dynamics (Fig 4A). The presence of sidebands constitutes a signature of frequency modulation, but frequency modulation theory can more specifically predict that the modulator frequency $M$ corresponds to the distance between non-harmonic sidebands (Fig 4B, inset). The magnitude and number of significant sidebands around the fundamental frequency could be obtained by deriving Bessel functions of the Fourier series (Boashash 2016). However, Carson's rule (Boashash 2016) can be used to approximate the bandwidth of significant sidebands as $2(D+M)=0.02$, which provides a good approximation of frequency modulation properties in our predator-prey system (Fig 4B, inset).

\section{Application to a large-scale metacommunity}

We tested the robustness of phase asynchrony to the previous assumptions of small (3 patch) metacommunities, weak spatial coupling, and relaxation oscillators. We used a spatially-continuous (integro-differential) predator-prey model previously applied to spatiotemporal dynamics of large-scale coastal ecosystems (Gouhier et al. 2010). This model corresponds to an infinite linear array of equally-spaced communities, each governed by Rosenzweig-MacArthur dynamics $d X_{i} / d t=F\left(X_{i}\right)$ as in (1). Spatial coupling differed slightly from (1) in that coupling strength decreases exponentially with linear distance between communities. Distance-independent coupling is unrealistic for systems of this spatial extent.

To relax the assumptions of weak coupling and relaxation oscillators, we decreased the separation of temporal scales between predators and preys with $\epsilon=0.7$, and by increasing coupling to $\delta=0.1$. The resulting parameters were those used to validate short-term spatial synchrony with data of Mytilus californianus (California mussel) distribution over 1800km along the North East Pacific coast (Gouhier et al. 2010). We then tested for the presence of frequency modulation associated with validated spatiotemporal patterns characterized by spatial periodicity in phase coherence (Gouhier et al. 2010).

We simulated the integro-differential equations with periodic boundary conditions over a time period of $T=12000$ and kept the last 3000 time steps of each simulation output for analysis. 
We extracted phase from local time series and calculated local instantaneous period using the same method as for our 3-patch simulations.

Results produce complex spatiotemporal patterns (Fig 5A) reported in previous studies (Gouhier et al. 2010), which lead to similarly complex spatiotemporal modulation of frequency over an extended spatial domain (Fig 5B). More specifically, large-scale aggregations of high frequency oscillations over the 1D domain explain the spatial periodicity in short-term phase coherence found in both numerical simulations and data of Mytilus from 1999-2007 (Gouhier et al. 2010). Over longer temporal scales, the distribution of these high frequency patches becomes dynamic, but lead to qualitatively similar regularity of local frequency modulation found in 3-patch networks (Fig 5C). We also analyzed the spatially-continuous model with the same parameters that were used for our 3 patch model in figures 3 and $4 \mathrm{~B}$, with $\eta=0.15$ and $\delta=10^{-4}$. Weak coupling and relaxation oscillators also predict frequency modulations (Fig 5C), but of much smaller amplitude than non-relaxation oscillators under strong coupling. These results suggest that frequency modulation is robust and even promoted by strongly coupled regular oscillators.

\section{Discussion}

Our study shows that spatial phase asynchrony, defined as the lack of a fixed phase difference among fluctuating populations, can be driven by weak dispersal in a homogeneous predator-prey metacommunity with a small (3-patch) or large (infinite) number of patches. This endogenous phase asynchrony results in the emergence of a collective frequency modulation across the metacommunity. Frequency modulation is distinct from exogenous asynchrony that can arise from environmental heterogeneity, and can be detected from individual time series. Dispersal-induced asynchrony reveals a new mechanism for the maintenance of heterogeneity and non-stationarity driven by species interactions and movement. The detection of long-term fluctuations in frequency remains challenging with existing ecological datasets. However, the emergence of frequency modulation could lead to the development of statistical tools for the inference of intrinsic and extrinsic causes of spatiotemporal heterogeneity from local (non-spatial) time series. Phase asynchrony and frequency modulation are compatible with spatiotemporal patterns of spatial synchrony previously observed in marine metacommunities, and have important implications for predicting population persistence and community stability in spatially structured habitats.

\section{Frequency modulation and the emergence of ecological asynchrony}

The loss of phase synchrony is central to predicting non-stationary properties of large-scale ecosystems, including their stability and productivity. Phase asynchrony can result from environmental heterogeneity in natural frequencies of populations (Blasius et al. 1999, Koelle \& Vandermeer 2005) (Fig 1C,D). Given no or weak dispersal, these heterogeneous frequencies can be maintained and lead to continuous changes in phase differences across the metacommunity. Uncorrelated environmental fluctuations can also cause heterogeneity in the frequency and phase 
difference of communities (Allstadt et al. 2015). Frequency modulation can be viewed as a special case where a periodic fluctuation imposes a periodic change in local frequencies (Fig 1E,F). If the frequency modulation is spatially heterogeneous, it can cause phase asynchrony. Our results reveal that frequency modulation can emerge from weakly coupled homogeneous oscillations and is associated with phase asynchrony in the absence of any environmental heterogeneity. When compared to the environmental forcing of heterogeneous frequencies, one important implication of dispersal-driven frequency modulation is the non-stationarity of both local and regional time series, which can be used to infer spatial dynamics from local time series.

Frequency modulation is a common feature of natural and synthesized sounds, with many applications in signal processing (Boashash 2016). It has a long history in engineering to encode and decode complex signals into its carrier (the single-patch predator-prey orbit in the present study), and a modulator prescribing the rate and magnitude of frequency modulation to the carrier (Fig 1E,F). In ecology, frequency modulation has been applied to ecological and behavioral studies of sound production by individuals (Morton 1975, Truax 2001). Its general spectral signature (in the frequency domain) is the presence of non-harmonic sidebands around harmonic peaks. The distribution of these sidebands depends on the frequency and amplitude of the modulator relative to the carrier signal. In that context, frequency modulation is usually considered an exogenous cause of phase asynchrony, where modulator signals force the carrier oscillation. Our results show that frequency modulation and the frequency modulator signal itself can be emerging properties of metacommunity dynamics. It is therefore possible to extract the modulator signal and infer regional phase asynchrony from local time series. Applying frequency modulation theory to our simulation results, we show how the spacing of non-harmonic sidebands (Fig 4B inset) from the local time series provides a good approximation of the regional frequency modulation signal. This result contributes an important tool to infer not only the emergence of dispersal-driven phase asynchrony, but also the temporal scales of both local and regional dynamics.

\section{Scaling up from species traits to metacommunities}

Ecological synchrony can help scaling up from individual traits to the dynamics of metacommunities. For example, phase synchrony can be predicted from individual traits such as birth, death and consumption rates that respond to environmental heterogeneity (Blasius et al. 1999, Koelle \& Vandermeer 2005, Goldwyn \& Hastings 2009). Increasing the difference between predator and prey growth leads to relaxation-type oscillations that are more prone to phase-locked synchrony than regular oscillations in 2-patch metacommunities (Goldwyn \& Hastings 2007). While 2-patch predator-prey systems display a limited range of spatial dynamics, larger ecological networks can exhibit very high complexity, including long transient (Ruxton \& Doebeli 1996, Hastings 2001, Cazelles et al. 2001) and chaotic (Holland \& Hastings 2008) dynamics. One approach to resolving this complexity consists in studying the emergence of dynamical behaviour in networks of intermediate size (Marleau et al. 2014). Our results show that a qualitatively dif- 
ferent dynamical behaviour, phase asynchrony through frequency modulation, can emerge from a minimal increase of spatial complexity, from 2 to 3 discrete habitats. Our results thus support the importance of pulse-relaxation oscillations as an important dynamical behavior for predicting spatial synchrony in weakly-coupled communities. Future studies should elucidate the role of pulse-relaxation oscillations and the robustness of phase asynchrony to more complex species interaction networks than predator-prey interactions, and to heterogeneous spatial networks.

Phase asynchrony leads to non-stationarity of individual predator-prey time series over local (frequency and phase difference) and regional (averaging effect on frequency doubling and amplitude dampening) spatial scales. Inferring dispersal-induced asynchrony from such complex time series can be guided by properties of the embedded frequency modulator. The periodic modulation of frequencies reported here for a small network provides an important foundation for understanding complex dynamics over larger networks. In marine coastal ecosystems for example, the spatial dynamics of benthic invertebrates was predicted over regional scales using metacommunity models where local predator-prey fluctuations are strongly coupled by the passive transport of pelagic larvae (Gouhier et al. 2010). Predictions of dispersal-induced heterogeneity over large spatial networks $(>100 \mathrm{~km})$ were tested using spatially extensive but short-term data (Gouhier et al. 2010). Here we show that the same model also predicts dispersal-induced temporal non-stationarity, with long-term oscillations in the frequency of local population fluctuations (Guichard \& Gouhier 2014), similarly to our analysis of 3-patch networks. Frequency modulation constitutes a novel ecological mechanism linking species interactions and movement to the maintenance of spatiotemporal heterogeneity across scales, with important implications for linking metacommunity theory and data (Grainger \& Gilbert 2016).

\section{Inferring metacommunity dynamics from time series}

Inferring endogenous and exogenous causes of variability from time series has been a long standing goal of population and community ecology (Jassby \& Powell 1990, Louca \& Doebeli 2014, Cavanaugh et al. 2013). Detecting and interpreting spatial synchrony has proven especially challenging because most periodic time series also contain a strong stochastic component (Turchin 2003), in which case statistical correlation and phase synchrony can become confounded (Haydon \& Greenwood 2000, Gouhier \& Guichard 2014). Also, in the presence of long transient dynamics (Holland \& Hastings 2008, Hastings 2004), resulting non-stationarity of spatial synchrony and of the period of fluctuations can be confounded with dispersal-induced phase asynchrony reported here. Non-stationarity in the period of ecological fluctuations and in spatial synchrony has been detected in a number of long-term time series of small mammal populations (Henden et al. 2009, Cazelles et al. 2008), and much progress has been made towards the statistical detection of such time-dependent frequency dynamics (Cazelles et al. 2008). However, non-stationary periods and synchrony are interpreted as the result of environmental drivers, through their spatial and/or temporal heterogeneity (e.g. spatially uncorrelated temporal fluctuations; Allstadt et al. 2015, Arumugam et al. 2015). Dispersal-induced frequency modulation provides a new interpretation 
for non-stationary frequency dynamics in ecological time series. We predict such modulation in the absence of any environmental heterogeneity using a spatially-continuous metacommunity model applied to the regional dynamics of predator-prey fluctuations coupled by dispersal (Guichard \& Gouhier 2014). These predictions were previously tested in a natural coastal ecosystem using short-term but spatially-extensive data on spatial synchrony of marine invertebrates (Gouhier et al. 2010). By predicting spatial and temporal signatures of dispersal-induced frequency fluctuations, our theory of endogenous asynchrony offers a method to infer ecological processes by using temporal or spatial data as a proxy for complex spatial dynamics. However, the availability of long-term ecological time series is still limited to a few natural systems. Also, more efforts are needed to develop inference methods based on a combination of shorter-term spatial and temporal data, in order to assess the relative importance of abiotic and biotic drivers of frequency modulation.

Phase asynchrony contributes to a more general theory of ecological synchrony by defining endogenous and exogenous mechanisms causing the loss of phase synchrony, and by characterizing its dynamical and statistical properties. Phase synchrony provides an equilibrium theory (phase locking) of non-equilibrium population dynamics. Phase asynchrony extends this theory to non-equilibrium dynamics of spatial heterogeneity (phase difference) itself. Because phaseasynchronous time series contain characteristic statistical signatures across spatial and temporal scales, phase asynchrony improves the robustness of current inference frameworks used for studying the causes of non-stationary spatiotemporal fluctuations in natural ecosystems. 


\section{References}

Abbott, K. C. (2011), 'A dispersal-induced paradox: synchrony and stability in stochastic metapopulations', Ecol. Lett. 14(11), 1158-1169.

Allstadt, A. J., Liebhold, A. M., Johnson, D. M., Davis, R. E. \& Haynes, K. J. (2015), 'Temporal variation in the synchrony of weather and its consequences for spatiotemporal population dynamics', Ecology 96(11), 2935-2946.

Arumugam, R., Dutta, P. S. \& Banerjee, T. (2015), 'Dispersal-induced synchrony, temporal stability, and clustering in a mean-field coupled Rosenzweig-MacArthur model', Chaos 25(10), 10.1063/1.4933300.

Ashwin, P., King, G. \& Swift, J. W. (1990), 'Three identical oscillators with symmetric coupling', Nonlinearity 3(3), 585-601.

Baesens, C., Guckenheimer, J., Kim, S. \& MacKay, R. (1991), 'Three coupled oscillators: modelocking, global bifurcations and toroidal chaos', Physica D: Nonlinear Phenom. 49(3), 387-475.

Bjørnstad, O. N., Ims, R. A. \& Lambin, X. (1999), 'Spatial population dynamics: analyzing patterns and processes of population synchrony', Trends Ecol. Evol. 14(11), 427-432.

Blasius, B., Huppert, A. \& Stone, L. (1999), 'Complex dynamics and phase synchronization in spatially extended ecological systems', Nature 399, 354-359.

Boashash, B. (2016), Time-frequency signal analysis and processing, second edn, Elsevier.

Cavanaugh, K. C., Kendall, B. E., Siegel, D. A., Reed, D. C., Alberto, F. \& Assis, J. (2013), 'Synchrony in dynamics of giant kelp forests is driven by both local recruitment and regional environmental controls', Ecology 94(2), 499-509.

Cazelles, B., Bottani, S. \& Stone, L. (2001), 'Unexpected coherence and conservation', Proc. R. Soc. B 268(1485), 2595-2602.

Cazelles, B. \& Boudjema, G. (2001), 'The Moran effect and phase synchronization in complex spatial community dynamics', Am. Nat. 157(6), 670-676.

Cazelles, B., Chavez, M., Berteaux, D., Ménard, F., Vik, J. O., Jenouvrier, S. \& Stenseth, N. C. (2008), 'Wavelet analysis of ecological time series', Oecol. 156(2), 287-304.

Emelianova, Y. P., Kuznetsov, A., Sataev, I. \& Turukina, L. (2013), 'Synchronization and multifrequency oscillations in the low-dimensional chain of the self-oscillators', Physica D: Nonlinear Phenom. 244(1), 36-49.

Goldwyn, E. E. \& Hastings, A. (2007), 'When can dispersal synchronize populations?', Theor. Pop. Biol. 73, 395-402.

Goldwyn, E. E. \& Hastings, A. (2009), 'Small heterogeneity has large effects on synchronization of ecological oscillators', Bull. Math. Biol. 71(1), 130-144.

Goldwyn, E. E. \& Hastings, A. (2011), 'The roles of the Moran affect and dispersal in synchronizing oscillating populations', J. Theor. Biol. 289, 237-246.

Gouhier, T. C. \& Guichard, F. (2014), 'Synchrony: quantifying variability in space and time', Meth. Ecol. Evol. 5(6), 524-533. 
Gouhier, T. C., Guichard, F. \& Menge, B. A. (2010), 'Ecological processes can synchronize marine population dynamics over continental scales', Proc Natl Acad Sci U S A 107(18), 8281-6.

Grainger, T. N. \& Gilbert, B. (2016), 'Dispersal and diversity in experimental metacommunities: linking theory and practice', Oikos 125(9), 1213-1223.

Guichard, F. \& Gouhier, T. C. (2014), 'Non-equilibrium spatial dynamics of ecosystems', Math. Biosci. 255, 1-10.

Hastings, A. (2001), 'Transient dynamics and persistence of ecological systems', Ecol. Lett. 4, 215-220.

Hastings, A. (2004), 'Transients: the key to long-term ecological understanding?', Trends Ecol. Evol. 19(1), 39-45.

Haydon, D. T. \& Greenwood, P. E. (2000), 'Spatial coupling in cyclic population dynamics: models and data', Theor. Pop. Biol. 58(3), 239-254.

Henden, J.-A., Ims, R. A. \& Yoccoz, N. G. (2009), 'Nonstationary spatio-temporal small rodent dynamics: evidence from long-term norwegian fox bounty data', J. An. Ecol. 78(3), 636-645.

Holland, M. D. \& Hastings, A. (2008), 'Strong effect of dispersal network structure on ecological dynamics', Nature 456(7223), 792-794.

Hoppensteadt, F. C. \& Izhikevech, E. M. (1997), Weakly connected neural networks, Springer, New York.

Hoppensteadt, F. C. \& Izhikevich, E. M. (1998), 'Thalamo-cortical interactions modeled by weakly connected oscillators: could the brain use fm radio principles?', Biosystems 48, 85-94.

Jansen, V. A. \& de Roos, A. (2000), The Role of Space in Reducing PredatorPrey Cycles. In: The Geometry of Ecological Interactions: Simplifying Spatial Complexity, eds, Cambridge University Press, pp. 183-201.

Jassby, A. D. \& Powell, T. M. (1990), 'Detecting changes in ecological time series', Ecology pp. 2044-2052.

Kim, S., Kook, H., Lee, S. G. \& Park, M.-H. (1998), 'Synchronization and clustering in a network of three globally coupled neural oscillators', Int. J. Bif. Chaos 8(04), 731-739.

Koelle, K. \& Vandermeer, J. (2005), 'Dispersal-induced desynchronization: from metapopulations to metacommunities', Ecol. Lett. 8(2), 167-175.

Lampert, A. \& Hastings, A. (2016), 'Stability and distribution of predator-prey systems: local and regional mechanisms and patterns', Ecol. Lett. 19(3), 279-288.

Lande, Engen \& Sæther (1999), 'Spatial scale of population synchrony: Environmental correlation versus dispersal and density regulation', Am. Nat. 154(3), 271-281.

Liebhold, A., Koenig, W. D. \& Bjørnstad, O. N. (2004), 'Spatial synchrony in population dynamics', Annu. Rev. Ecol. Evol. Syst. 35, 467-490.

Louca, S. \& Doebeli, M. (2014), 'Distinguishing intrinsic limit cycles from forced oscillations in ecological time series', Theor. Ecol. 7(4), 381-390.

Marleau, J. N., Guichard, F. \& Loreau, M. (2014), 'Meta-ecosystem dynamics and functioning on finite spatial networks', Proc. R. Soc. B 281(1777), 20132094. 
Montbrió, E., Kurths, J. \& Blasius, B. (2004), 'Synchronization of two interacting populations of oscillators', Phys. Rev. E 70, 056125.

Morton, E. S. (1975), 'Ecological sources of selection on avian sounds', Am. Nat. 109, 17-34.

Ruxton, G. \& Doebeli, M. (1996), 'Spatial self-organization and persistence of transients in a metapopulation model', Proc. R. Soc. B 263(1374), 1153-1158.

Truax, B. (2001), 'Handbook of acoustic ecology', Comp. Mus. J. 25, 93-94.

Turchin, P. (2003), Complex population dynamics: a theoretical/empirical synthesis, Vol. 35 of Monograph s in population biology, Princeton University Press.

Wall, E., Guichard, F. \& Humphries, A. R. (2013), 'Synchronization in ecological systems by weak dispersal coupling with time delay', Theor. Ecol. 6(4), 405-418.

Zhang, Y., Lutscher, F. \& Guichard, F. (2015), 'How robust is dispersal-induced spatial synchrony?', Chaos 25(036402), 10.1063/1.4906951. 


\section{List of Figures}

1 Examples of time series of abundance (A,C,E) and of corresponding frequency $(\mathrm{B}, \mathrm{D}, \mathrm{F})$ representing phase synchrony $(\mathrm{A}, \mathrm{B})$ and phase asynchrony driven by either spatially heterogeneous habitats $(\mathrm{C}, \mathrm{D})$ or by temporal frequency modulation $(\mathrm{E}, \mathrm{F})$. When habitat heterogeneity imposes differences in local frequencies of population fluctuations (D), the phase difference between time series grows continuously $(\mathrm{C})$. When time series result from frequency modulation (E), they can be decomposed, or demodulated, into $(i)$ the original single patch predator-prey orbit called the carrier (not shown), and (ii) the modulator signal corresponding to the time series of oscillating frequency $(\mathrm{F})$. The properties of the modulated time series (E) then depend of the frequency deviation (ratio of the observed frequency to the frequency of a single patch) and magnitude of the modulator signal

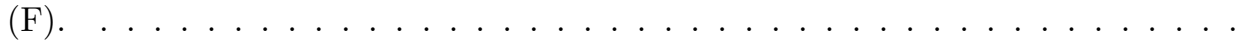

2 Bifurcation diagrams of $\psi$ (phase difference) from phase equations (A) and from full model simulations (B) with $\delta=10^{-5}$ and as $\eta$ varies in $(0.15,0.4)$. (A) shows a schematic bifurcation diagram of the phase-difference dynamics (6). The straight horizontal lines correspond to the all-in-phase state at $(0,0)$ and the travelling wave state at $(2 \pi / 3,2 \pi / 3)$; the curves represent amplitudes of limit cycles. Thicker lines are stable objects, thin lines unstable. Letters $\mathrm{H}$ and $\mathrm{SH}$ indicate the Hopf and the subcritical Hopf bifurcation. SNL stands for the saddle-node bifurcation of limit cycles. The vertical lines correspond to the values of $\eta$ for which the phase plane schematic is given in Figure A.2. (B) Symbols correspond to local stability for each semi-random initial condition scenario: $<1 \%$ deviation from in-phase (filled diamonds), $<1 \%$ deviation from homogeneous phase locking at $2 \pi / 3$ (red squares), and $10 \%$ deviation from in-phase (filled circles). . . . . . . . . .

3 Local and regional dynamics of system (1) under phase asynchrony $(\eta=0.15, \delta=$ $\left.10^{-4}\right)$. (A) Time series of local abundance in each patch (colored lines) and of regional (average over 3 patches, black line). (B) Time series of phase difference $(\psi)$ for each pair of patches. (C) Time series of frequency deviation (ratio of observed to single patch frequency) of oscillations in each patch (relative to frequency of the single orbit). . . . . . . . . . . . . . . . . . .

4 Power spectrum of local predator abundance time series for $(\mathrm{A})$ the single-patch (uncoupled) orbit and (B) phase asynchronous metacommunity dynamics. Parameter values as in Fig 3. In (A), only a single patch is considered, which constitute the 'carrier' (unmodulated) signal with a fundamental frequency of 0.94 cycles/t. In (B), random initial phases lead to phase asynchrony, which produces a frequency-modulated signal. The inset shows the non-harmonic sidebands around the distribution fundamental frequency at $\approx 0.1$ with sideband spacing of $\approx 7.10^{-4}$. 21 
5 Results from simulations of the 1D continuous-space predator-prey model. Spatiotemporal patterns of $(\mathrm{A})$ predator abundance and of $(\mathrm{B})$ the period deviation of oscillations (expressed as the ratio of observed to single patch period) for paramter values used in (Gouhier et al. 2010). (C) Local time series of the period deviation of predator abundance at a single location obtained from simulations displayed in (A-B; solid line), and for a simulation with parameters corresponding to assumptions of our 3-patch analysis (dotted line) . . . . . . . . . . . . . . . . . 22 

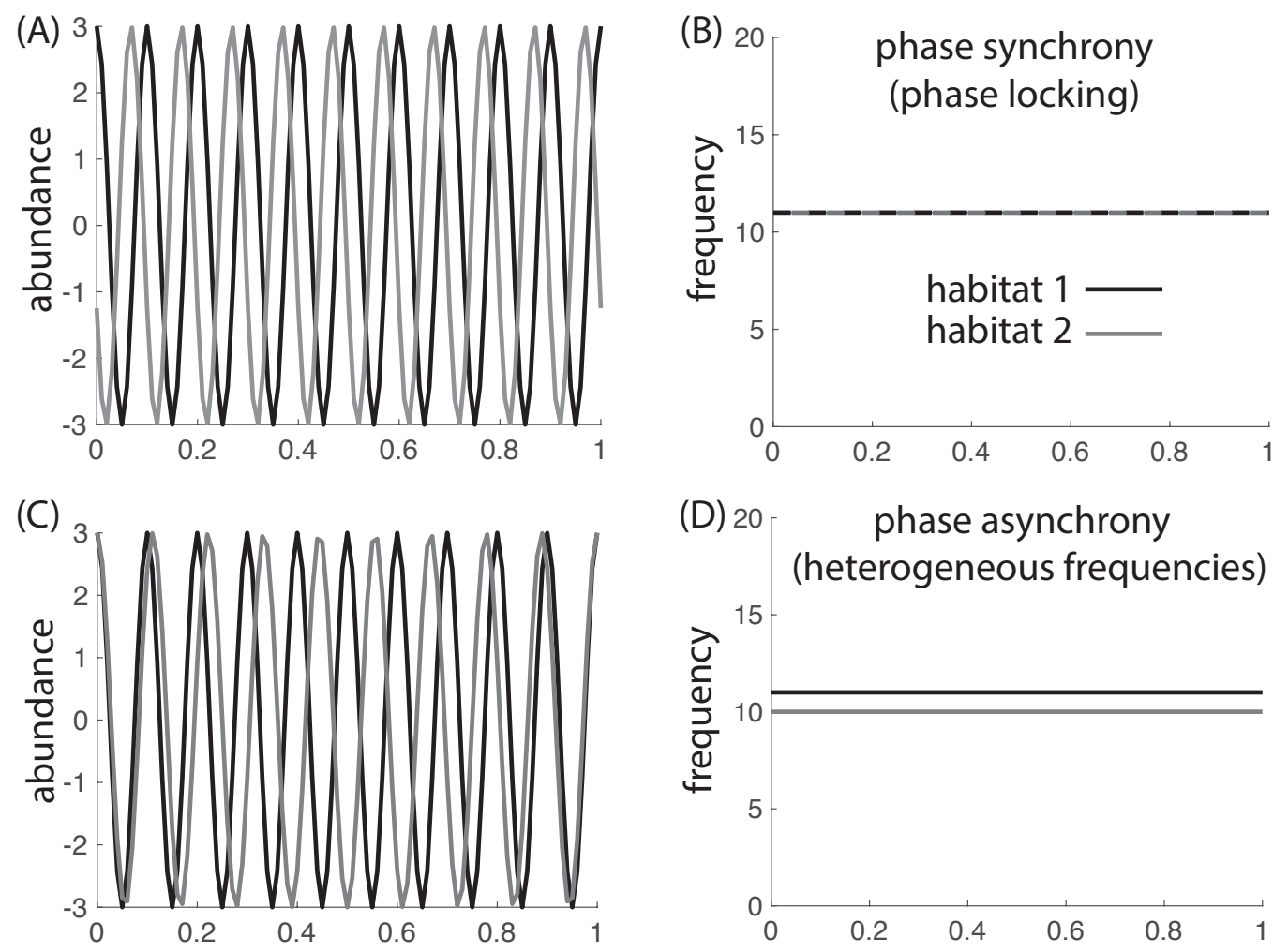

(D) $20\left[\begin{array}{c}\text { phase asynchrony } \\ \text { (heterogeneous frequencies) }\end{array}\right.$
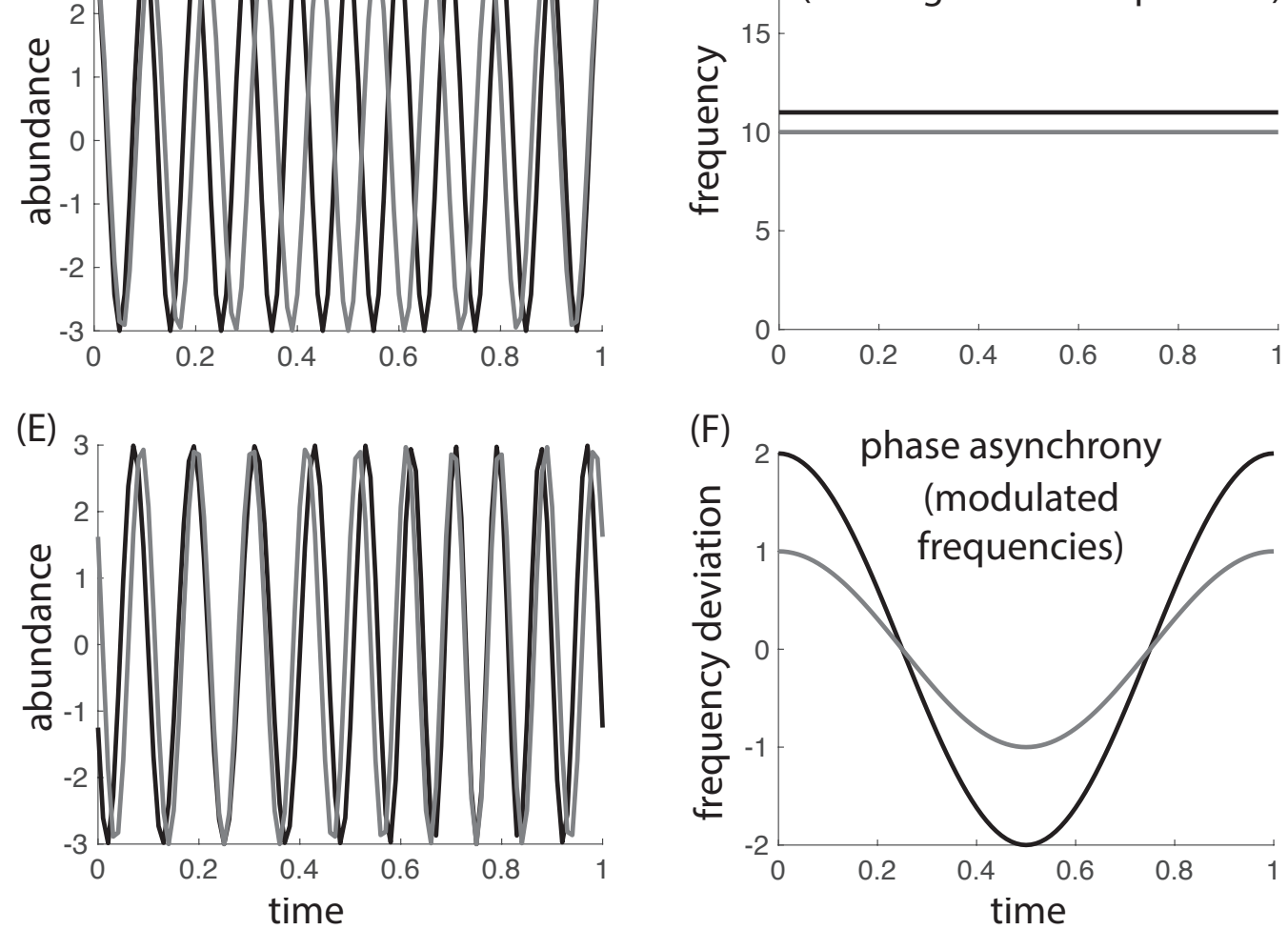

Fig. 1 


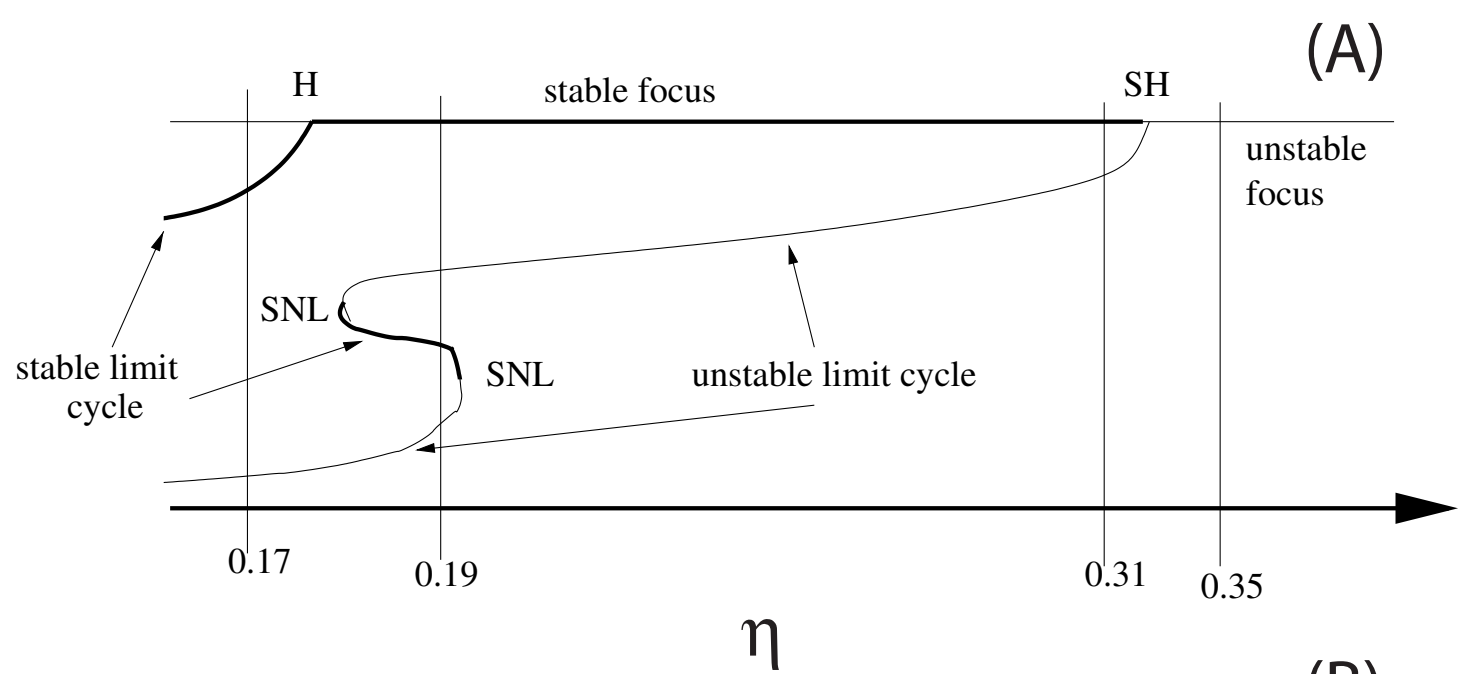

(B)

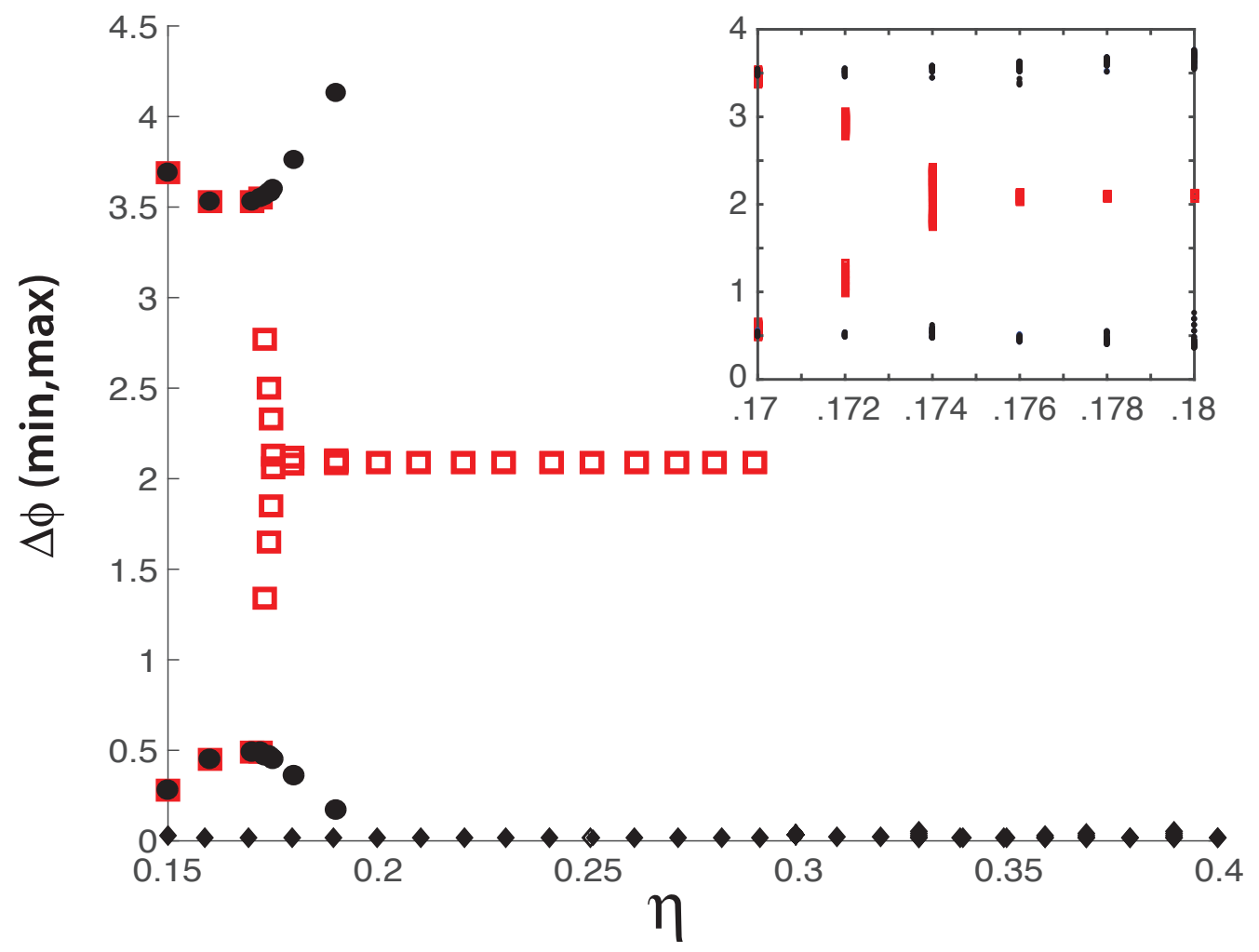

Fig. 2 


\section{(A)}
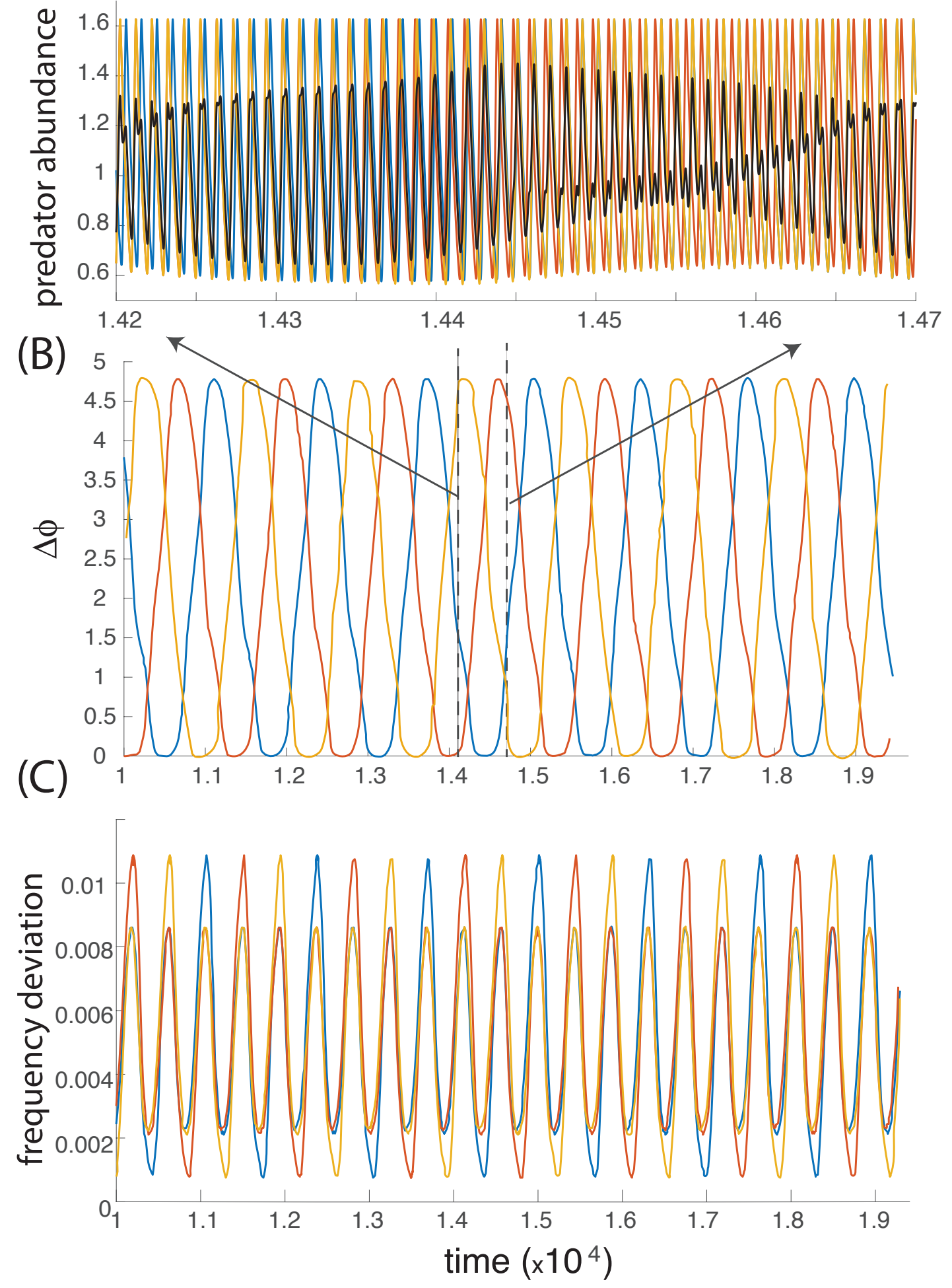

Fig. 3 

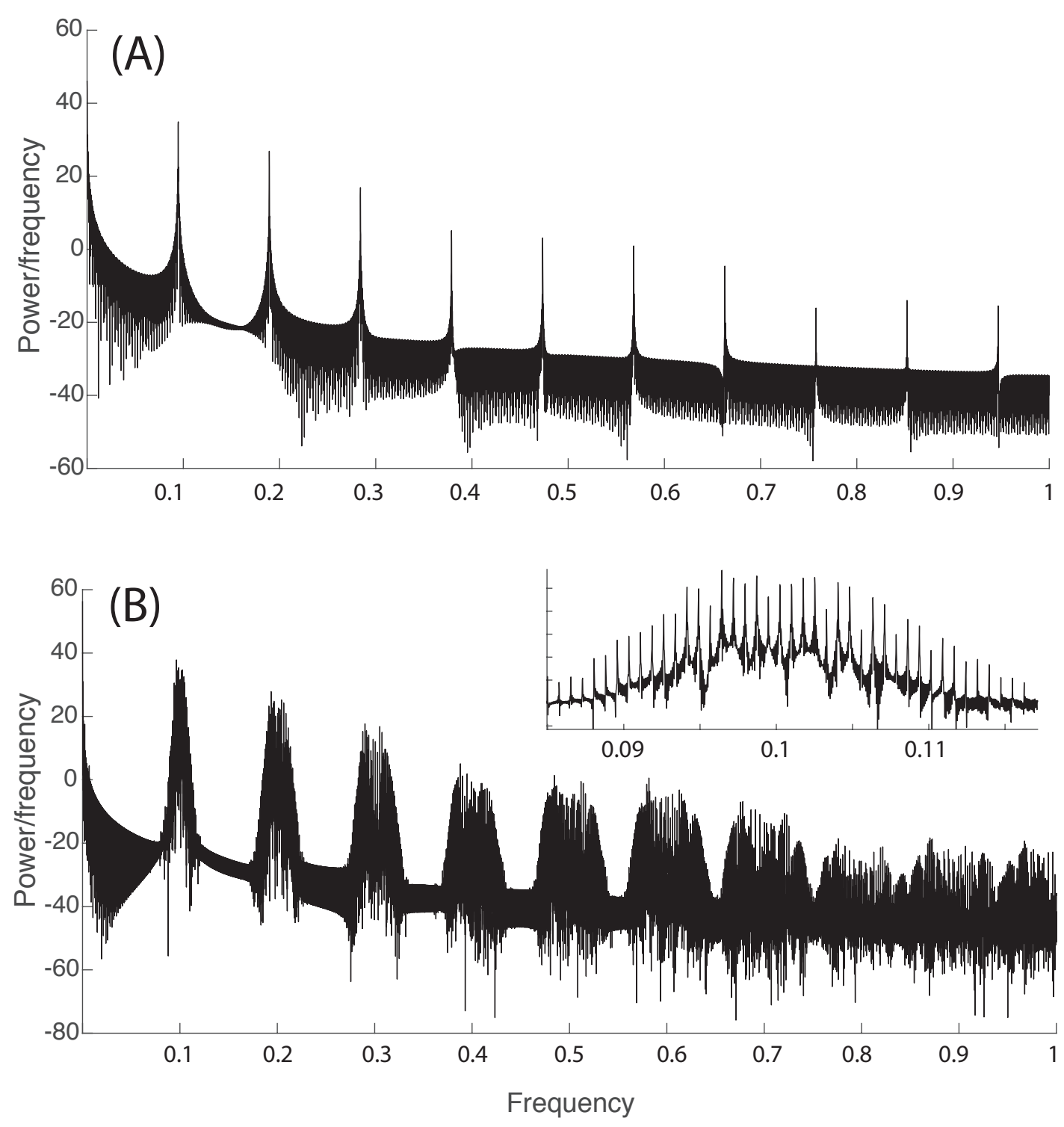

Fig. 4 

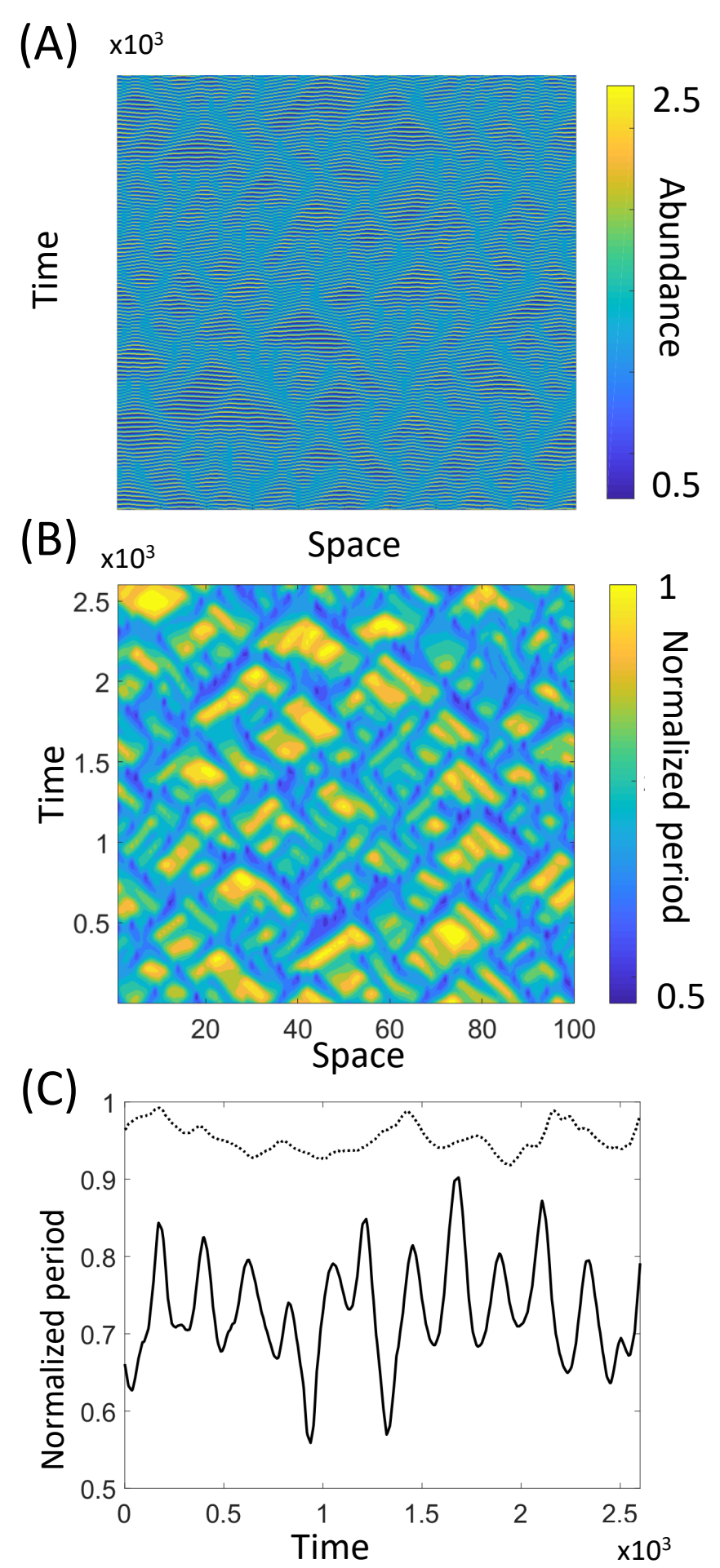

Fig. 5 


\section{A Appendix - Model Analysis}

Here, we provide details on the qualitative analysis of the planar dynamical system given by the phase-difference equations (6), i.e.

$$
\begin{aligned}
\frac{d \psi_{1}}{d t} & =\frac{1}{2}\left[H\left(-\psi_{1}\right)+H\left(\psi_{2}\right)-H\left(\psi_{1}\right)-H\left(\psi_{1}+\psi_{2}\right)\right], \\
\frac{d \psi_{2}}{d t} & =\frac{1}{2}\left[H\left(-\psi_{1}-\psi_{2}\right)+H\left(-\psi_{2}\right)-H\left(-\psi_{1}\right)-H\left(\psi_{2}\right)\right] .
\end{aligned}
$$

The most important ingredient of these equations is the $2 \pi$-periodic function $H$. It is related to the so-called infinitesimal phase-response curve, $\hat{\gamma}$, along a periodic orbit (Goldwyn \& Hastings 2007). The infinitesimal phaseresponse curve is the solution of the differential equation

$$
\frac{d \hat{\gamma}}{d t}=-D F(\gamma(t)) \times \hat{\gamma}
$$

with the normalization condition $\hat{\gamma}(t) \times \gamma^{\prime}(t)=1$. In other words, $\hat{\gamma}$ is given by the linearization equation of the vectorfield $F$ around the stable periodic orbit $\gamma$. With this notation, function $H$ is given by

$$
H(x)=\frac{1}{T} \int_{0}^{T} \hat{\gamma}(t) \cdot(\gamma(t+x / \Omega)-\gamma(t)) d t
$$

where $T$ is the period of the periodic orbit $\gamma(t)$ of the Rosenzweig-MacArthur model and $\Omega=2 \pi / T$ is the frequency.

Even though function $H$ is not explicitly given, we can still proceed with the qualitative analysis of the phasedifference system by finding steady states and calculating their stability conditions. Periodicity of the function $H$ is an important aspect in almost all the considerations to follow.

\section{Zero phase-differences}

The 'all-in-phase' state $\left(\psi_{1}, \psi_{2}\right)=(0,0)$ is a steady-state solution, independent of parameter values. Hence, all-in-phase synchrony is always possible. Moreover, this state is always locally asymptotically stable: if a metacommunity is initially close to in-phase synchrony, it will converge to in-phase synchrony. To see that $(0,0)$ is locally stable, we linearize (A.1) and get the Jacobi matrix

$$
J(0,0)=\frac{H^{\prime}(0)}{2}\left(\begin{array}{cc}
-3 & 0 \\
0 & -3
\end{array}\right) .
$$

Thus, the local stability of $(0,0)$ is determined by the sign of $H^{\prime}(0)$. If $H^{\prime}(0)>0\left(H^{\prime}(0)<0\right)$, the all-in-phase steady state is linearly stable (unstable). This condition is exactly the same as for the two-patch model studied in (Goldwyn \& Hastings 2007, Zhang et al. 2015). Based on those results, the all-in-phase state will be locally stable for all parameter sets that we study here.

\section{Symmetry}

As we look for further steady states, we note that the phase difference equations are $2 \pi$-periodic and possess several symmetries. In particular, if $\left(\psi_{1}^{*}, \psi_{2}^{*}\right)$ is a steady state of system (A.1), then the following are also steady states: $\left(2 \pi+\psi_{1}^{*}, \psi_{2}^{*}\right),\left(\psi_{1}^{*}, 2 \pi+\psi_{2}^{*}\right),\left(2 \pi+\psi_{1}^{*}, 2 \pi+\psi_{2}^{*}\right)$ and $\left(2 \pi-\psi_{2}^{*}, 2 \pi-\psi_{1}^{*}\right)$. In fact, the vector field in (A.1) on the square $[0,2 \pi) \times[0,2 \pi)$ is symmetric with respect to the diagonal $y=2 \pi-x$. It can therefore be completely represented by its value on the triangle $0 \leq \psi_{1}+\psi_{2} \leq 2 \pi$. The schematic representations in Figure A.2 only show one such triangle for each case. The computationally generated phase-plane plots in Figure A.3 show the entire square $[0,2 \pi) \times[0,2 \pi)$ and illustrate the symmetry along the diagonal. 


\section{Equal phase differences}

The next particular steady-state solution that we investigate has equal, non-zero phase differences between all three oscillators. Such a state is known as a 'travelling-wave state' (Goldwyn \& Hastings 2011) a 'rotating wave' (Ashwin et al. 1990) or 'splay state'. If we assume that $\left(\psi_{1}^{*}, \psi_{2}^{*}\right)=\left(x^{*}, x^{*}\right)$ is such a state, we have the equations:

$$
\begin{aligned}
& 0=H\left(-x^{*}\right)+H\left(x^{*}\right)-H\left(x^{*}\right)-H\left(2 x^{*}\right), \\
& 0=H\left(-2 x^{*}\right)+H\left(-x^{*}\right)-H\left(-x^{*}\right)-H\left(x^{*}\right) .
\end{aligned}
$$

Hence, if $2 x^{*}=-x^{*}$ modulo $2 \pi$ then these equations are satisfied. Therefore, travelling-wave states with phase difference $x^{*}=2 \pi / 3$ or $x^{*}=4 \pi / 3$ exist independently of parameter values in the system.

Knowing the stability behaviour of travelling-wave states will turn out crucial to understanding the dynamics of the system. The Jacobi matrix at the state $\left(x^{*}, x^{*}\right)=(2 \pi / 3,2 \pi / 3)$ has the form

$$
J=\frac{1}{2}\left(\begin{array}{cc}
-2 H^{\prime}\left(2 x^{*}\right)-H^{\prime}\left(x^{*}\right) & H^{\prime}\left(x^{*}\right)-H^{\prime}\left(2 x^{*}\right) \\
H^{\prime}\left(2 x^{*}\right)-H^{\prime}\left(x^{*}\right) & -H^{\prime}\left(2 x^{*}\right)-2 H^{\prime}\left(x^{*}\right)
\end{array}\right) .
$$

To get this form, we used the fact that for $x^{*}=2 \pi / 3$ we have $2 x^{*}=-x^{*} \bmod 2 \pi$ and that $H$ is $2 \pi$-periodic. We calculate the trace and determinant of this matrix as

$$
\operatorname{tr} J=-3\left(H^{\prime}\left(x^{*}\right)+H^{\prime}\left(2 x^{*}\right)\right), \quad \operatorname{det} J=3\left[H^{\prime}\left(x^{*}\right)^{2}+H^{\prime}\left(2 x^{*}\right)^{2}+H^{\prime}\left(x^{*}\right) H^{\prime}\left(2 x^{*}\right)\right] .
$$

To evaluate the eigenvalues of $J$, we calculate the discriminant as

$$
(\operatorname{tr} J)^{2}-4 \operatorname{det} J=-3\left(H^{\prime}\left(x^{*}\right)-H^{\prime}\left(2 x^{*}\right)\right)^{2} \leq 0 .
$$

Hence, the eigenvalues are not real (unless $H^{\prime}\left(x^{*}\right)=H^{\prime}\left(2 x^{*}\right)$ ) and therefore, the point is a focus or spiral. This observation also follows from general symmetry considerations (Ashwin et al. 1990). The stability is then given by the sign of the trace of $J$. In particular, the travelling-wave state is stable if $\operatorname{tr} J<0$ or

$$
H^{\prime}\left(x^{*}\right)+H^{\prime}\left(2 x^{*}\right)>0 .
$$

We evaluate this quantity numerically for our parameter set. The plot in Figure A.1 shows that the travelling-wave state is stable for intermediate values $\eta$ but unstable for small and for large values.

Fig. A.1: Numerical evaluation of the stability condition (A.6) for our default parameter set. The travelling-wave state is stable when the quantity in the figure is positive.

\section{2-in-phase states}

Finally, we will see steady-state solutions where one phase difference is zero, so-called '2-in-phase states' (Ashwin et al. 1990), for example $\left(\psi_{1}^{*}, \psi_{2}^{*}\right)=\left(y^{*}, 0\right)$. These arise when $y^{*}$ satisfies

$$
2 H\left(y^{*}\right)=H\left(-y^{*}\right)
$$

These states are located in an invariant set. In fact, if $\psi_{2}=0$ then $d \psi_{2} / d t=0$. Hence, if the phase difference $\psi_{2}$ is zero initially, then it will be zero for all times. In that case, the dynamics reduce to the line $\left(\psi_{1}, 0\right)$, with $0 \leq \psi_{1} \leq 2 \pi$. The two end-points are locally stable, as we have seen above when studying the stability of $(0,0)$. Since the dynamics are one-dimensional, there has to be at least one steady state on the line with $0<\psi_{1}<2 \pi$, and if there is only one it has to be unstable. Hence, the 2-in-phase state always exists. By symmetry, the same reasoning holds for $\psi_{1}=0$ and there have to be at least three such states, namely $\left(y^{*}, 0\right),\left(2 \pi-y^{*}, y^{*}\right)$, and $\left(0,2 \pi-y^{*}\right)$. 


\section{Stability changes}

In the corresponding 2-patch system, the anti-phase locked solution changes stability, and additional out-of-phaselocked solutions appeared, when parameters are varied in such a way that the relative temporal scales between different processes differed (Goldwyn \& Hastings 2007, Zhang et al. 2015, Wall et al. 2013). We focus on how the dynamics of (??) change as parameter $\eta$ decreases. We describe several scenarios in Figure A.2 (schematic) and Figure A.3 (actual) and summarize the results in a bifurcation diagram (Figure A.4, see also Figure 2 in the main text). For large values of $\eta \geq 0.32$ the travelling-wave state is an unstable focus; all non-constant solutions converge to the all-in-phase locked state. As $\eta$ decreases, the travelling-wave state becomes stable through a subcritical Hopf bifurcation and an unstable limit cycle emerges. Continuing to decrease $\eta$, two additional limit cycles appear in a saddle-node bifurcation so that there are three limit cycles now. The middle one is stable (e.g. $\eta=0.19$ ). The two inner limit cycles eventually collide and disappear in another saddle-node bifurcation of limit cycles (near $\eta=0.175$ ). The travelling-wave state undergoes another Hopf bifurcation, this time supercritical, so that a stable limit cycle emerges $(\eta=0.17)$. The stable limit cycles correspond to solutions that are not phase locked and hence show true asynchrony.

The situation is similar to the generic bifurcation diagram for three identical, weakly coupled oscillators in (Ashwin et al. 1990). Those authors mention that the branch that emerges from the (subcritical) Hopf bifurcation can "fold back on itself and create saddle-node bifurcations of tori". What we do not observe in our parameter range is the change of stability of the all-in-phase locked state that is required for the global bifurcation that these authors observed. 


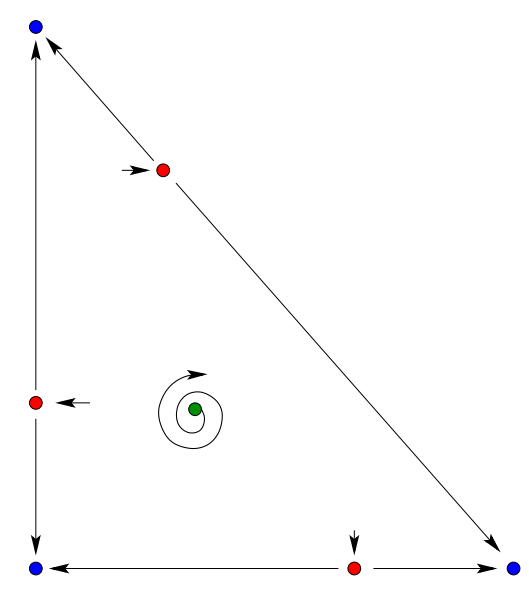

(a) $\eta=0.35$

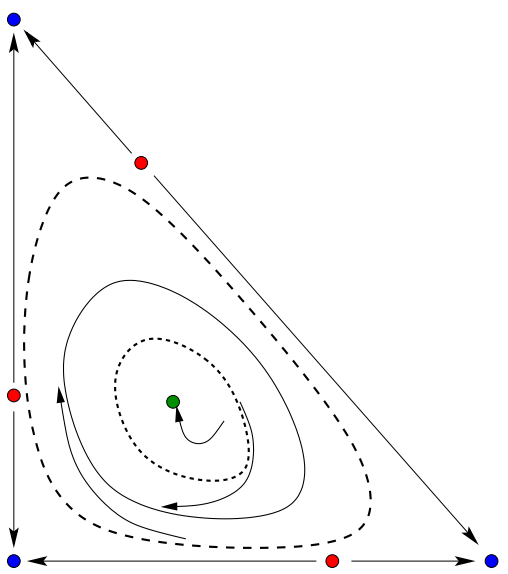

(c) $\eta=0.19$

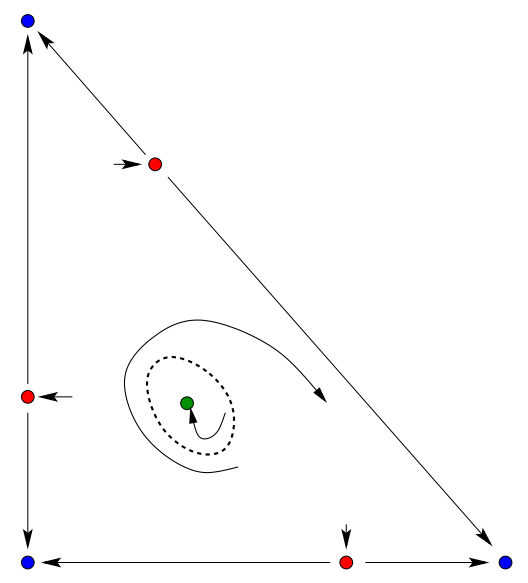

(b) $\eta=0.31$

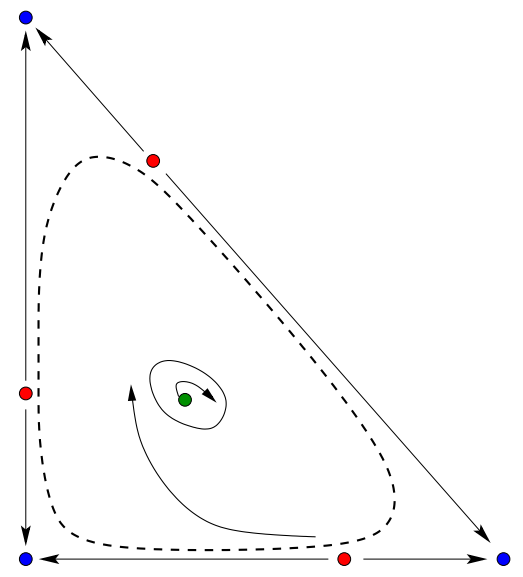

(d) $\eta=0.17$

Fig. A.2: Schematic of the phase plane of system (6) for $0.15<\eta<0.4$. Blue dots correspond to the all-in-phase locked state and are locally stable for all values. Red dots correspond to 2-in-phase states and are saddles. The green dot stands for the travelling-wave state. The latter state is an unstable spiral for $\eta \geq 0.35$ (top left). After a subcritical Hopf bifurcation, it becomes stable with a surrounding unstable limit cycle $(\eta=0.31$, top right). After the first saddle-node bifurcation, there are three limit cycles of which the inner and outer are unstable $(\eta=0.19$, bottom left). After the second saddle-node bifurcation, there is only a large unstable limit cycle (no plot). Then a supercritical Hopf bifurcation generates a stable limit cycle and the travellingwave state becomes unstable again $(\eta=0.17$, bottom right). The other parameter values are $\epsilon=0.1$, and $\alpha=0.4$. 


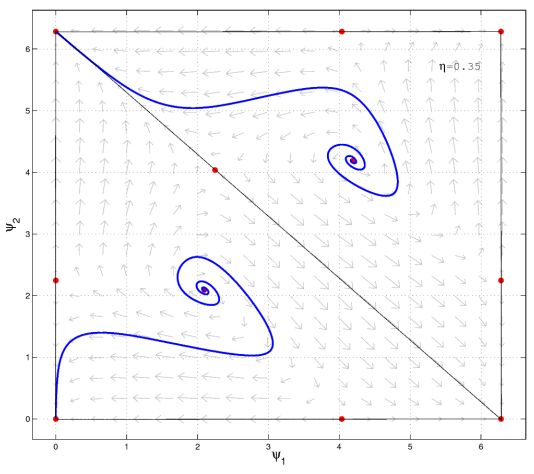

(a) $\eta=0.35$

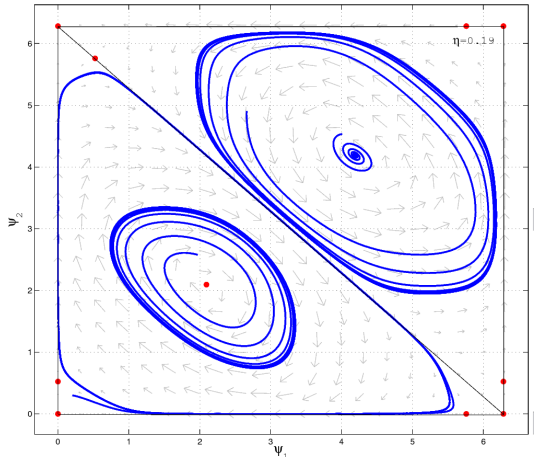

(c) $\eta=0.19$

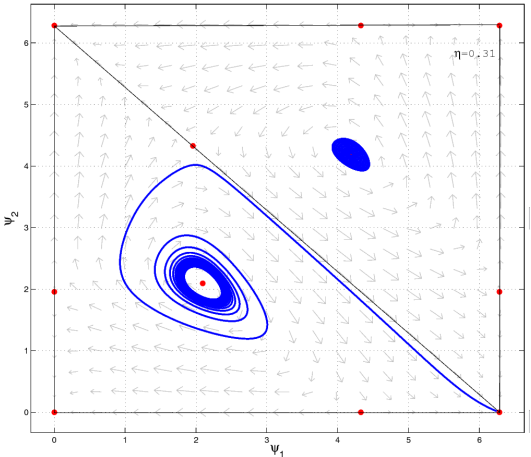

(b) $\eta=0.31$

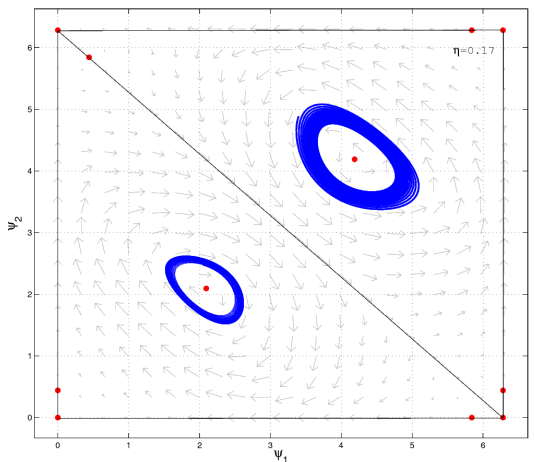

(d) $\eta=0.17$

Fig. A.3: Phase planes of system (6) for different values of $\eta$, corresponding to Figure A.2. Plot (a) shows two complete trajectories, connecting the unstable travelling-wave state to the stable all-inphase state. Plot (b) also shows two complete orbits, one located inside the unstable limit cycle, one outside. The upper right triangle in plot (c) shows how two forward trajectories approach two stable objects: the travelling-wave state and the stable periodic orbit of asynchronous phase. The lower left triangle in plot (c) shows how two backwards orbits approach the two unstable limit cycles. Plot (d) highlights the single stable limit cycle as it is approached from the exterior (top right triangle) and the interior (bottom left triangle). The large unstable is extremely difficult to capture because it is so close to the axes that numerical accuracy becomes an issue. 


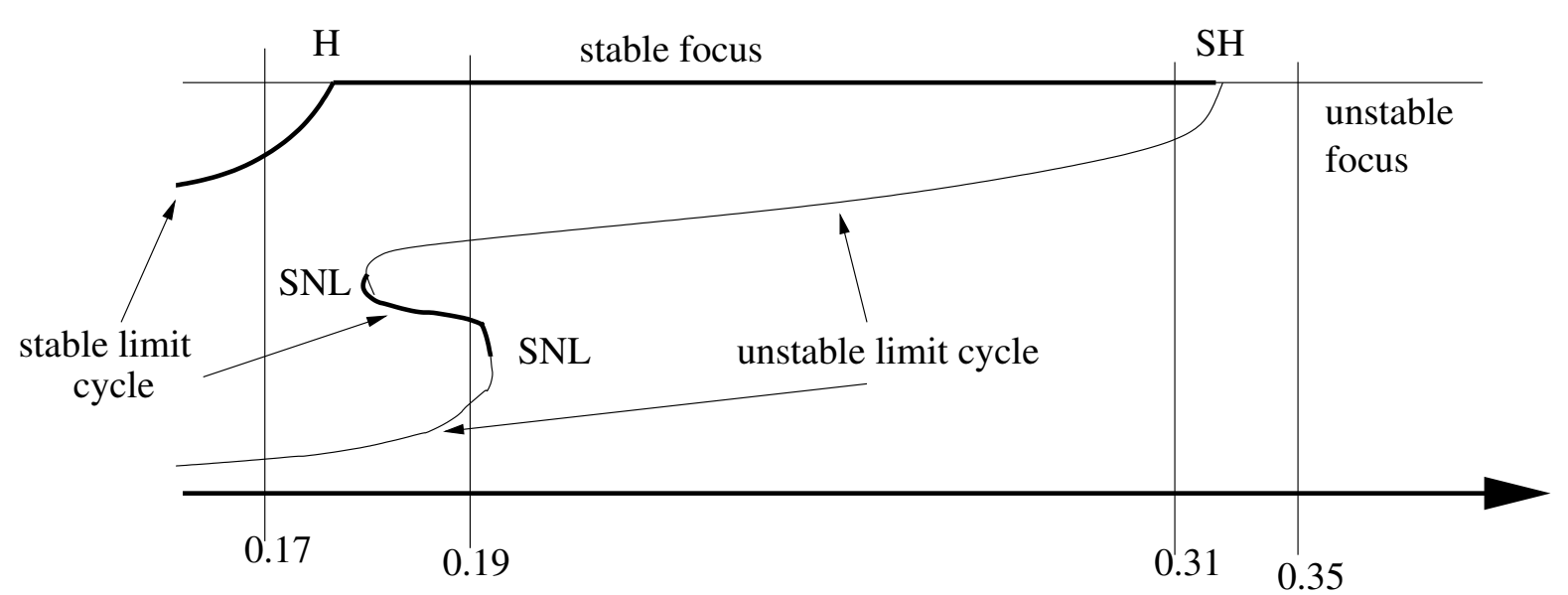

Fig. A.4: Schematic bifurcation diagram of the phase-difference dynamics (6) as $\eta$ varies in $(0.15,0.4)$. The straight horizontal lines correspond to the tall-in-phase state at $(0,0)$ and the travelling-wave state at $(2 \pi / 3,2 \pi / 3)$; the curves represent amplitudes of limit cycles. Thicker lines are stable objects, thin lines unstable. Letters $\mathrm{H}$ and SH indicate the Hopf and the subcritical Hopf bifurcation. SNL stands for the saddle-node bifurcation of limit cycles. The vertical lines correspond to the values of $\eta$ for which the phase plane schematic is given in Figure A.2 